\section{Equivalência transcultural de três escalas utilizadas para estimar a aptidão cardiorrespiratória: estudo em idosos}

\author{
Cross-cultural equivalence of three scales used to \\ estimate cardiorespiratory fitness in the elderly
}

\author{
${ }_{1}^{1}$ Instituto de Medicina \\ Social, Universidade do \\ Estado do Rio de Janeiro, \\ Rio de Janeiro, Brasil. \\ 2 Instituto de Educação Física \\ e Desportos, Universidade do \\ Estado do Rio de Janeiro, \\ Rio de Janeiro, Brasil. \\ 3 Programa de Pós-graduação \\ em Ciências da Atividade \\ Física, Universidade Salgado \\ de Oliveira, Niterói, Brasil. \\ Correspondência \\ G. A. Maranhão Neto \\ Departamento de \\ Epidemiologia, Instituto \\ de Medicina Social \\ Universidade do Estado do \\ Rio de Janeiro. \\ Rua São Francisco Xavier \\ 524, Rio de Janeiro, $R J$ \\ 20559-900, Brasil. \\ maranhaoneto@gmail.com
}

\begin{abstract}
This study aimed at establishing the cross-cultural equivalence of scales used to evaluate physical activity level and measure cardiorespiratory fitness, for further application in elderly subjects. Three scales were identified after systematic review: Veterans Physical Activity Questionnaire (VSAQ), Rating of Perceived Capacity (RPC), and Physical Activity Rating (PA-R). The model proposed by Herdman et al. was applied to analyze equivalence. Test-retest reliability was calculated in a sample of 12 elderly subjects (74.5 \pm 3.5 years) using Lin's concordance coefficient and intraclass correlation coefficient. Good reproducibility was detected in all scales except RPC. Due to the small sample size, hardly any conclusions can de drawn, but the results point to the need for changes in the original scale's structures. The findings also suggest the adequacy of the scale's Portuguese-language version, although further validity studies appear to be necessary.
\end{abstract}

Physical Fitness; Health of the Elderly; Reproducibility of Results
Geraldo de Albuquerque Maranhão Neto 1,2

Antonio Carlos Monteiro Ponce de Leon 1

Paulo de Tarso Veras Farinatti 2,3

\section{Introdução}

Durante as últimas duas décadas, baixos níveis de aptidão cardiorrespiratória, indicador da capacidade dos sistemas cardiovascular e respiratório de fornecer oxigênio durante uma atividade física contínua 1, têm sido associados a maior morbi-mortalidade. Essas associações são comparáveis às de outros indicadores de saúde, tais como massa corporal, pressão arterial, níveis de colesterol sanguíneo e tabagismo 1,2,3,4,5,6,7.

Apesar de sua importância, a utilização da medida da aptidão cardiorrespiratória em estudos epidemiológicos é limitada, devido ao seu elevado custo de aferição em termos de recursos materiais e humanos 8,9. Entretanto, estratégias alternativas de aferição da aptidão cardiorrespiratória têm sido propostas e suas propriedades avaliadas em alguns estudos 10,11. Uma delas consiste em estimar a aptidão cardiorrespiratória sem a necessidade da realização de testes de exercício. No lugar de exercícios, equações de regressão múltipla que contemplam características físicas e hábitos de vida dos indivíduos são usadas para estimar os níveis de aptidão cardiorrespiratória, o que torna esta estratégia uma opção viável na pesquisa epidemiológica, por conta da implementação simples e menos onerosa 10,11,12.

Está bem estabelecido na literatura que a redução da aptidão cardiorrespiratória é decorrente do processo natural de envelhecimento 13,14 . Mas, quanto maior esta diminuição, maior 
o risco de uma série de agravos de saúde causados por doenças hipocinéticas, bem como a perda da autonomia física, maior dependência e necessidade de cuidados clínicos 15,16. Desenvolver formas de aferição da aptidão cardiorrespiratória na população é, portanto, uma questão relevante de pesquisa. Como a menor capacidade funcional e a fragilidade muscular dos idosos demandam muitos cuidados na realização de testes físicos, esta abordagem fica dificultada em estudos epidemiológicos. Assim, justifica-se a necessidade de desenvolver equações sem exercício específicas para a população de idosos 17,18,19,20,21. A literatura internacional não apresenta modelos específicos para avaliar a aptidão cardiorrespiratória nessa população, tampouco existem iniciativas nacionais para encontrar alternativas mais simples para a mensuração da aptidão cardiorrespiratória, apesar de a principal indicação para a realização de testes ergométricos em idosos no Brasil ser a avaliação da aptidão cardiorrespiratória 22 .

Para o desenvolvimento de uma nova equação sem exercícios, é preciso considerar as características dos modelos existentes, a fim de usar estratégias já utilizadas e bem sucedidas. Uma das principais variáveis incluídas nas estimativas da aptidão cardiorrespiratória sem testes de exercícios é a informação sobre o nível de atividade física, o que é verificado por meio de escalas que podem remeter tanto ao histórico de atividades 23,24 , quanto à autopercepção do condicionamento físico 25,26 . Ao contrário das medidas mais objetivas como massa corporal e estatura, a medida referente à atividade física deve passar por adaptações que atendam diferenças sócioculturais ou de idioma, para que possa ser aplicada em populações de características diferentes daquelas em que foi validada.

A grande maioria das escalas utilizadas para estimar a aptidão cardiorrespiratória foi formulada na língua inglesa e por isso, direcionada à população de países anglófonos. Para a superação dessa limitação na aplicação desse tipo de escala em países com cultura diversa, duas estratégias podem ser utilizadas: o desenvolvimento de novas escalas ou a modificação e adaptação de escalas previamente validadas, valendo-se de um processo de adaptação cultural 27 .

A primeira opção demanda uma quantidade maior de tempo e de recursos humanos e financeiros. Para a criação de um novo instrumento, deve-se adequar e/ou propor novos conceitos, elaborar e selecionar itens que com eles guardem coerência teórica e, enfim, testar a validade do instrumento. Contudo, é questionável a necessidade de se criarem novas escalas, caso já existam outras com a mesma proposta e boa qualidade.
Nesse contexto, justifica-se uma adaptação das versões originais das escalas utilizadas para estimar a aptidão cardiorrespiratória. Não basta, no entanto, que tais instrumentos sejam simplesmente traduzidos; torna-se necessária também uma avaliação rigorosa de sua tradução, além de uma adaptação transcultural.

Assim, o objetivo do presente estudo foi realizar a equivalência transcultural de escalas empregadas para a avaliação do nível de atividade física e que são utilizadas como estimativas da aptidão cardiorrespiratória, para posterior utilização em indivíduos idosos.

\section{Método}

\section{Seleção das escalas}

Para a seleção das escalas, realizou-se uma revisão sistemática de estudos publicados cujo objetivo era estimar a aptidão cardiorrespiratória sem a utilização de testes de exercício e, em seguida, as escalas utilizadas por esses estudos foram examinadas. As estratégias de busca foram as consideradas em artigo publicado em Cadernos de Saúde Pública, em 2004 28. Este processo resultou na seleção de 32 estudos, todos publicados como artigos originais entre os anos de 1971 e 2007. Desses, 15 relataram a inclusão de indivíduos tendo idade de 60 anos ou mais na amostra, não representando necessariamente a maior parte da amostra. Todos que abrangeram pessoas idosas na amostra incluíram alguma informação sobre atividade física, com apenas dois não relatando o uso de escalas.

Os seguintes critérios de inclusão foram utilizados para selecionar as escalas: utilização nos estudos que tenham apresentado as amostras mais numerosas de indivíduos com idade a partir de 60 anos e gerado as equações com as melhores estimativas, além da freqüência do relato do uso da escala nos artigos selecionados. De acordo com esses critérios, as seguintes escalas foram selecionadas:

- Veterans SpecificActivity Questionnaire(VSAQ) 25,26,29: inicialmente usada com indivíduos com idade média de 62 anos, sendo bastante utilizada na determinação de protocolos para testes ergométricos mas não utilizada em estudos epidemiológicos. Trata-se de uma escala de atividades físicas de intensidade progressiva com escores que vão de 1 a 13, em que o respondente assinala qual das atividades listadas lhe causaria fadiga ou falta de ar durante a realização;

- Rating of Perceived Capacity (RPC) 30: uma adaptação do VSAQ que apresenta uma lista de atividades com escores que vão de 1 a 20 de acor- 
do com a intensidade, devendo ser assinalada a atividade que se é capaz de realizar por um período mínimo de trinta minutos. Para os criadores dessa escala, a adaptação do VSAQ fazia-se necessária em virtude de várias pessoas terem apresentado dificuldades para selecionar a atividade apropriada, especialmente quando se tratava das atividades localizadas na parte intermediária da lista (intensidade moderada). $\mathrm{O}$ fato de o RPC ser uma adaptação de uma escala selecionada, desenvolvida com o intuito de facilitar a compreensão da escala original pela inclusão de atividades comuns a diversas culturas, justificou a sua inclusão no processo de equivalência transcultural.

O segundo critério de inclusão (estar presente no maior número de artigos) levou à adoção da Physical Activity Rating (PA-R) 23,24,31,32,33,34,35 como outra escala selecionada. Trata-se de uma escala progressiva com escores de 0 a 7 , de acordo com a intensidade das atividades apresentadas, em que deve ser selecionado o escore mais adequado de acordo com o histórico de atividades físicas dos últimos trinta dias.

\section{Equivalência transcultural das escalas}

Para esse processo, foi empregado o modelo proposto por Herdman et al. 36 , uma sistemática que envolve um aprofundamento cronologicamente ordenado de vários subtipos de equivalência. De acordo com esse modelo, é necessário levar em conta ao menos seis dimensões de equivalência para que um instrumento seja adaptado adequadamente para outro idioma, a saber: conceitual entre os itens, semântica, operacional, de mensuração e funcional.

A primeira delas, equivalência conceitual, diz respeito à existência do mesmo conceito num contexto semelhante, nos dois grupos populacionais (aquele em que a escala foi desenvolvida e aquele em que vai ser aplicada). Essa investigação pode ser feita por meio de revisão da literatura, da consulta a especialistas e, em segmentos da população em geral, pelas técnicas qualitativas como grupos focais. Uma vez considerada adequada, as demais dimensões são pesquisadas ${ }^{36}$. Os conceitos em um instrumento são, em geral, investigados mediante perguntas ou itens que devem ser correspondentes nos dois idiomas (equivalência entre os itens). Esta equivalência é considerada presente quando os itens avaliados são considerados relevantes em ambas as culturas. Para a avaliação das equivalências conceitual entre os itens, foi formado um grupo de especialistas composto por mestres e doutores das áreas de educação física, fisiologia do exercício e epidemiologia.
Todos realizaram uma consulta prévia às escalas sob análise.

A equivalência semântica concerne à obtenção de um mesmo efeito causado pelo instrumento nos respondentes em diferentes idiomas. Sua obtenção é possível não apenas pela tradução literal, mas também da adaptação de certas palavras ou frases que tenham significado para a população em que a nova versão será aplicada. Inicialmente, para apreciação da equivalência semântica foi solicitada uma autorização aos criadores das escalas para que a tradução fosse realizada. Em seguida, a tradução de cada escala foi realizada por dois profissionais independentes, um deles com conhecimento na área de ciências do exercício e com diplomas de proficiência em idioma inglês nos seguintes testes: Test of English as a Foreign Language (TOEFL), Graduate Record Examinations Subject Test (GRE), International English Language Testing System (IELTS) e Test of Spoken English (TSE) e o outro profissional, um professor de inglês com graduação em letras e com diploma de IELTS. As duas versões traduzidas de cada escala foram mais uma vez analisadas pelo grupo de pesquisadores que participou da apreciação das equivalências conceitual e de itens. Uma versão em português adaptada de cada uma das três escalas foi, então, definida.

Após esse processo, as versões em português foram traduzidas para o inglês (backtranslation) por um terceiro tradutor independente que desconhecia as versões originais. Esse tradutor possuía os diplomas de proficiência TOEFL, GRE e TSE. As versões elaboradas foram mais uma vez apreciadas pelo grupo de pesquisadores e então enviadas aos pesquisadores que desenvolveram originalmente o PA-R, o VSAQ e o RPC (respectivamente, os doutores Andrew Jackson, Jonathan Myers e Anita Wisén) por correio eletrônico, para que estes avaliassem e propusessem sugestões. As modificações sugeridas pelos autores foram incorporadas e as versões finais em português foram, finalmente, elaboradas.

A adequação e pertinência de aspectos operacionais na nova cultura - forma de administração, número de opções de resposta etc. - também devem ser observadas (equivalência operacional). Nessa etapa, cada versão da escala foi aplicada em indivíduos idosos. Originalmente, apenas o VSAQ 25,26 é relatado como uma escala auto-aplicada, enquanto os outros estudos originais não esclarecem se as escalas foram autoaplicadas ou aplicadas por meio de entrevistas 7,9,23,30. Na seqüência, avaliam-se as propriedades psicométricas da nova versão (equivalência de mensuração), quando possível comparandoas com a versão original. Por último chega-se à equivalência funcional, uma síntese das demais 
formas de equivalência, e que permite afirmar se uma escala pode ser aplicada na nova população-alvo. Somente após examinar os diferentes aspectos de equivalência apontados acima, é possível declarar que está estabelecida a adaptação transcultural da nova versão da escala está estabelecida 36 .

\section{Equivalência psicométrica das escalas}

Para a apreciação da equivalência de mensuração das escalas, geralmente recomenda-se que medidas de confiabilidade e validade sejam realizadas. No presente estudo, analisou-se apenas a confiabilidade. A análise da confiabilidade de uma escala compreende dois aspectos: a consistência interna e a estabilidade (confiabilidade teste-reteste) 36 . Uma escala apresenta alta consistência interna se cada um dos seus itens se correlaciona significativamente com a pontuação total. Esse atributo pode ser avaliado pelo coeficiente alpha de Cronbach. As escalas aqui estudadas possibilitam apenas uma resposta, não apresentando mais de um item, logo não há como calcular a consistência interna. Já a confiabilidade teste-reteste é mensurada quando a escala é aplicada em duas ocasiões distintas. Resultados próximos entre escores obtidos nas duas ocasiões sugerem boa reprodutibilidade da escala. A verificação da confiabilidade teste-reteste é um aspecto importante no desenvolvimento de novos instrumentos, mas infelizmente este tipo de análise não foi realizado nas versões originais das escalas, fato que impossibilitou a comparação da confiabilidade entre as versões original e traduzida, bem como criou, sem dúvida, uma lacuna que deve ser preenchida. Para a análise teste-reteste foram calculados os coeficientes de concordância de Lin (CCL) e de correlação intraclasse (CCI) entre os escores das escalas obtidos em um intervalo de duas semanas.

O CCL 37 foi desenvolvido com o objetivo de validar novos instrumentos e consiste em comparar as medidas do novo instrumento com as geradas por métodos já consagrados. Entretanto, o procedimento de cálculo do CCL também permite avaliar a concordância entre dois pares de medidas de uma mesma amostra em tempos diferentes. Neste contexto, se duas séries de medidas, analisadas para a verificação dos erros aleatórios e sistemáticos, apresentarem valores muito próximos ou idênticos, quando em um gráfico de dispersão, obter-se-á uma reta de perfeita concordância entre os pontos, com inclinação de $45^{\circ}$. O CCL compara a concordância entre os pares das medidas, medindo a variação em torno da linha de $45^{\circ}$. Uma vantagem do CCL é que se pode utilizar um mínimo de dez pares de medidas, que podem ser livres de distribuição. Alguns autores recomendam utilizar essa medida como complemento do CCI ${ }^{38}$. Já o CCI diferencia o componente de variabilidade que é atribuído ao erro das diferenças entre os dados. Tanto o CCL quanto o CCI têm a amplitude de variação de $0,00-1,00$. No presente estudo, a seguinte classificação foi adotada para ambos os coeficientes de concordância: 0,21-0,40 (fraca); 0,41-0,60 (moderada); 0,61-0,80 (substancial) e 0,81-1,00 (quase perfeita).

Para o estudo da confiabilidade teste-reteste, as escalas foram aplicadas a 12 sujeitos (11 mulheres e um homem) com idade média de $75 \pm 4$ anos, massa corporal de $60 \pm 10 \mathrm{~kg}$ e estatura de $1,6 \pm 0,07 \mathrm{~m}$. A massa corporal foi obtida em uma balança Filizola (Filizola Balanças Industriais S.A., São Paulo, Brasil) com precisão de um grama enquanto a estatura foi determinada em um estadiômetro de madeira com precisão de $0,1 \mathrm{~cm}$. Todos os avaliados estavam descalços e usavam roupas leves. Todos os participantes assinaram o Termo de Consentimento Livre e Esclarecido pósinformado, de acordo com as recomendações da convenção de Helsinki e da Resolução $n^{o}$. 196/96 do Conselho Nacional de Saúde do Brasil para pesquisas envolvendo seres humanos. $\mathrm{O}$ estudo foi aprovado pelo Comitê de Ética em Pesquisas do Instituto de Medicina Social, da Universidade do Estado do Rio de Janeiro (CEP/IMS/UERJ), com base no parecer emitido pelo relator no processo 0007.0.259.000-07.

\section{Resultados}

\section{Apreciação da equivalência conceitual e entre itens}

Com relação à equivalência conceitual, o grupo de pesquisadores concluiu que os conceitos estudados nos instrumentos originais seriam apropriados para utilização em nossa cultura, sugerindo inclusive que tanto a adaptação quanto a tradução das escalas seriam relativamente simples de serem realizadas. O mesmo grupo julgou que os itens das escalas necessitariam de poucas alterações em sua forma original ${ }^{36}$ (equivalência entre itens).

\section{Apreciação da equivalência semântica}

Ilustrando o processo de equivalência semântica, as duas traduções (T1 e T2) para cada escala e as versões finais (VF) são apresentadas nas Tabelas 1 a 6. Nelas, pode-se observar quando uma das versões foi escolhida com VF ou quando as adaptações foram realizadas a partir das duas versões. 
Alguns exemplos de alterações feitas para a VF: (1) no VSAQ (Tabelas 1 e 2) - a conversão e adaptação de unidades métricas (milhas para quilômetros) e de massa (libras para quilogra- mas), a exclusão da atividade "esquiar no campo (cross-country ski)" (escore 11), considerada de prática incomum em nossa cultura pelo grupo de especialistas; (2) no RPC (Tabelas 3 e 4) - a in-

Tabela 1

Etapas da equivalência semântica e versão final: Veteran Specific Activity Questionnaire (VSAQ).

Título T1(VF) Sublinhe a atividade que lhe causaria cansaço, falta de ar, desconforto no peito ou qualquer outra razão que o faça querer parar Mesmo que você não faça uma determinada atividade, tente imaginar como seria se você fizesse.

T2 Sublinhe a atividade que, se você realizar por um certo tempo, lhe causaria fadiga, falta de ar, desconforto no peito, ou qualquer outra razão que o faça querer parar. Mesmo se você não realiza uma atividade em particular tente imaginar como seria se você fizesse

Escore 1 VF Comer, vestir-se, trabalhar sentado (T1 = T2)

Escore 2 T1 Tomar uma ducha, fazer compras em shoppings e lojas de roupa, cozinhar Descer oito degraus (VF)

T2 Tomar uma ducha, fazer compras, cozinhar Descer oito degraus

Escore 3 T1 Andar devagar em uma superfície plana, por um ou dois quarteirões

Carregar compras, fazer serviços domésticos de intensidade moderada, como varrer o chão e passar o aspirador de pó

T2 Caminhar devagar por uma superfície plana por 1 ou 2 quarteirões

Trabalho doméstico de intensidade moderada, como passar o aspirador, varrer o chão ou carregar compras

VF Caminhar devagar em uma superfície plana por um ou dois quarteirões

Carregar compras, fazer serviços domésticos de intensidade moderada, como varrer o chão e passar o aspirador de pó

Escore 4 T1 Trabalho leve no quintal ou jardim, como juntar e colocar folhas numa sacola ou saco plástico, semear, varrer ou empurrar um cortador de grama a motor, pintura ou carpintaria leve (VF)

T2 Trabalho leve no quintal ou jardim, como varrer folhas ou empurrar um cortador de grama a motor/ pintura ou carpintaria leve

Escore 5 T1 Caminhar rápido/ Dança socialmente / Lavar o carro (VF)

T2 Caminhar rápido/ Dança de salão / Lavar o carro

Escore 6 VF Jogar golfe (nove buracos) carregando os próprios tacos (T1 = T2)

Carpintaria pesada, empurrar cortador de grama sem motor

Escore 7 T1 Carregar pesos com cerca de 60 libras, fazer trabalho pesado no exterior da casa, como cavar um buraco com pá, arar o solo, subir ladeira caminhando

T2 Carregar pesos com cerca de 60 libras, fazer trabalho pesado no exterior, como cavar, arar o solo, subir ladeira caminhando

VF Carregar pesos com cerca de $25 \mathrm{~kg}$, fazer trabalho pesado no exterior da casa, como cavar um buraco com pá, arar o solo, subir ladeira caminhando

Escore 8 T1(VF) Carregar sacolas de supermercado escada acima, mover móveis pesados. Corrida leve em superfície plana, subir escadas rapidamente T2 Carregar sacolas de supermercado escada acima, mover mobiliário pesado. Corrida leve, subir escadas rapidamente

Escore 9 VF Andar de bicicleta em ritmo moderado, serrar lenha, pular corda (devagar) (T1 = T2)

Escore 10 (T1 = T2) Natação acelerada, pedalar morro acima, correr a cerca de 6 milhas/hora VF Natação acelerada, pedalar morro acima, correr a cerca de $9,5 \mathrm{~km} / \mathrm{h}$

Escore 11 T1 Subir 2 lances de escada carregando algo pesado, como lenha ou uma criança no colo Andar de bicicleta em ritmo acelerado continuamente

Esquiar no campo (cross-country)

T2 Carregar algo pesado por 2 lances de escada, como uma criança ou lenha

Andar de bicicleta em ritmo acelerado continuamente

Esquiar no campo (cross-country)

VF Subir 2 lances de escada carregando algo pesado, como lenha ou uma criança no colo

Andar de bicicleta em ritmo acelerado continuamente

Escore 12 T1 Correr rápida e continuamente (plano horizontal, 8 minutos por milha)

T2 Correr rápida e continuamente (plano horizontal, oito minutos para cada 1,5 a 2,0km)

VF Correr rápida e continuamente (plano horizontal, 5 minutos para cada $1 \mathrm{~km}$ )

Escore 13 T1 Qualquer atividade física competitiva, incluindo aquelas com corrida acelerada (sprint) intermitente Correr, remar, ou pedalar de forma competitiva

T2 Qualquer atividade física competitiva, incluindo aquelas com corrida acelerada (sprint) intermitente

Correr de forma competitiva, remar de forma competitiva, pedalar de forma competitiva

VF Qualquer atividade física competitiva, incluindo aquelas com corrida acelerada (sprint) intermitente

Correr, remar, ou pedalar de forma competitiva 
Tabela 2

Questionário de atividades específicas para idosos (Veteran Specific Activity Questionnaire - VSAQ): versão final.

\begin{tabular}{|c|c|}
\hline MET & Atividades \\
\hline 1 & Comer, vestir-se, trabalhar sentado \\
\hline \multirow[t]{2}{*}{2} & Tomar uma ducha, fazer compras em shoppings e lojas de roupa, cozinhar \\
\hline & Descer oito degraus \\
\hline \multirow[t]{2}{*}{3} & Caminhar devagar em uma superfície plana, por um ou dois quarteirões \\
\hline & Carregar compras, fazer serviços domésticos de intensidade moderada, como varrer o chão e passar o aspirador de pó \\
\hline \multirow[t]{2}{*}{4} & $\begin{array}{l}\text { Trabalho leve no quintal ou jardim, como juntar e colocar folhas numa sacola ou saco plástico, semear, varrer ou empurrar um cortador de } \\
\text { grama a motor }\end{array}$ \\
\hline & Pintura ou carpintaria leve \\
\hline \multirow[t]{2}{*}{5} & Caminhar rápido \\
\hline & Dançar socialmente, lavar o carro \\
\hline \multirow[t]{2}{*}{6} & Jogar golfe (nove buracos) carregando os próprios tacos \\
\hline & Carpintaria pesada, empurrar cortador de grama sem motor \\
\hline \multirow[t]{2}{*}{7} & Subir ladeira caminhando, fazer trabalho pesado no exterior da casa, como cavar um buraco com pá, arar o solo \\
\hline & Carregar pesos com cerca de $25 \mathrm{~kg}$ \\
\hline \multirow[t]{2}{*}{8} & Mover móveis pesados \\
\hline & Corrida leve em superfície plana, subir escadas rapidamente, carregar sacolas de supermercado escada acima \\
\hline 9 & Andar de bicicleta em ritmo moderado, serrar lenha, pular corda (devagar) \\
\hline 10 & Natação acelerada, pedalar morro acima, andar rapidamente morro acima, correr a cerca de 9,5km/h \\
\hline \multirow[t]{2}{*}{11} & Subir 2 lances de escada carregando algo pesado, como lenha ou uma criança no colo \\
\hline & Andar de bicicleta em ritmo acelerado continuamente \\
\hline 12 & Correr rápida e continuamente (plano horizontal, 5 minutos para cada $1 \mathrm{~km}$ ) \\
\hline \multirow[t]{2}{*}{13} & Qualquer atividade física competitiva, incluindo aquelas com corrida acelerada (sprint) intermitente \\
\hline & Correr, remar, ou pedalar de forma competitiva \\
\hline
\end{tabular}

Nota: Sublinhe a atividade que lhe causaria cansaço, falta de ar, desconforto no peito ou qualquer outra razão que o faça querer parar. Mesmo que você não faça uma determinada atividade, tente imaginar como seria se você fizesse.

MET: unidade metabólica de repouso.

clusão do termo cooper juntamente com o termo "correr devagar" (escore 8), visto que ainda é um termo muito utilizado em nossa cultura, o RPC foi considerado o instrumento que apresentou atividades relevantes e aceitáveis em ambas as culturas e por isso, de mais fácil adaptação; (3) no PA-R (Tabelas 5 e 6) - a inclusão das atividades "caminhar para exercitar-se" e "hidroginástica" nos escores 2 e 3 , atividades consideradas mais comuns em nossa cultura e praticadas por indivíduos idosos, inserção das atividades "futebol" e "voleibol" referentes aos escores 4 a 7 , além da conversão e adaptação das unidades métricas e de massa.

Após a definição das versões finais em português e da retradução, as versões foram enviadas aos autores. Nesse último caso, apenas a Dra. Anita Wisén, responsável pelo desenvolvimento da escala RPC, sugeriu uma pequena modificação, qual seja, a retirada do termo: "mais de $15 \mathrm{~km} / \mathrm{h}$ " no quesito: "correr muito rápido" (escore 15). Segundo ela, vários respondentes estavam apresentando dificuldades para visualizar sua velocidade de corrida.

\section{Apreciação da equivalência operacional}

Como estratégia inicial, as três escalas foram autopreenchidas em uma seqüência aleatória por cinco idosos selecionados aleatoriamente entre os participantes de programa de atividades físicas desenvolvido pelo Laboratório de Atividade Física e Promoção da Saúde, do Instituto de Educação Física e Desportos da UERJ. Após o preenchimento, todos foram entrevistados sobre a facilidade de compreensão das escalas. Ao término do processo, as respostas foram analisadas pelo grupo de pesquisadores, que decidiu manter o formato impresso (layout, estrutura diagramática) como o das escalas originais. Foi decidido, entretanto, que os respondentes teriam acesso às escalas, mas elas seriam lidas e preenchidas para os idosos por um aplicador. Tal resolução justificou-se, pois os idosos apresentaram dificuldades 
Etapas da equivalência semântica e versão final: Rating of Perceived Capacity (RPC).

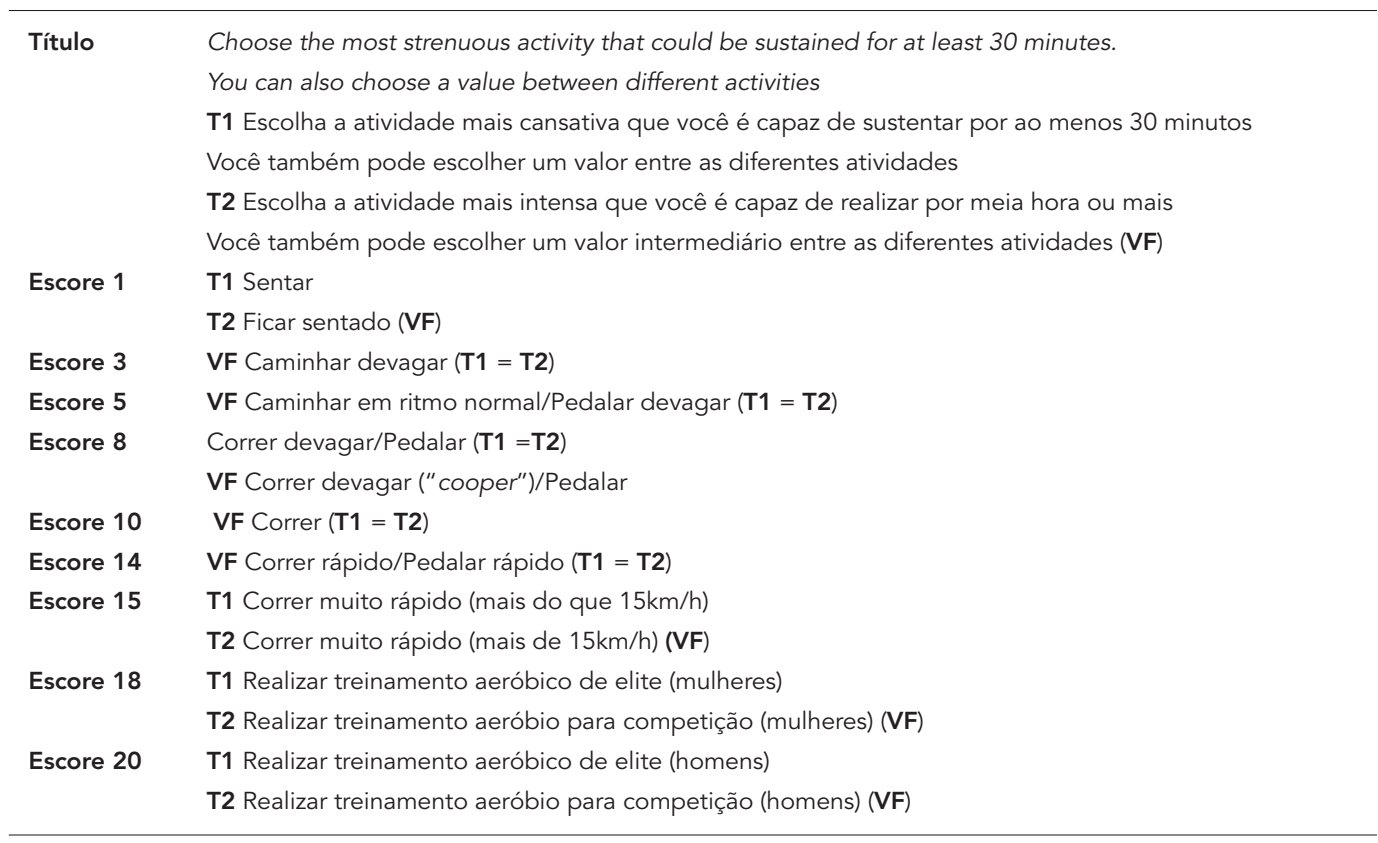

para visualizar as letras e constantemente perguntavam sobre como deveriam proceder. Após a decisão pela mudança da forma de aplicação das escalas, outros cinco idosos responderam as três escalas, com as mesmas sendo lidas e preenchidas pelo aplicador. Após o processo, novas entrevistas foram realizadas e nenhum deles teve dúvida sobre como proceder.

\section{Apreciação das equivalências de mensuração}

A Tabela 7 apresenta a estatística descritiva dos escores obtidos nas três escalas nos dois momentos em que foram aplicadas, além dos resultados das análises de confiabilidade teste-reteste.

Os valores encontrados para o CCL e CCI sugerem que o RPC teria uma menor confiabilidade em comparação com as demais escalas, com índices classificados como substanciais ou moderados (considerando-se o intervalo de confiança). OVSAQ e o PA-R, por outro lado, obtiveram índices considerados de moderados a quase perfeitos.

\section{Discussão}

Considerando que a equivalência funcional é uma síntese de todas as formas de equivalência, nas três novas versões das escalas ela se revelou

\section{Tabela 4}

Classificação de capacidade percebida (Rating of Perceived Capacity - RPC): versão final.

\section{Atividades}

Ficar sentado

Caminhar devagar

Caminhar em um ritmo normal/Pedalar devagar

Correr devagar ("cooper")/Pedalar

Correr

Correr rápido/Pedalar rápido

Correr muito rápido

Realizar treinamento aeróbio para competição (mulheres)

Realizar treinamento aeróbio para competição (homens)

Nota: escolha a atividade mais intensa que você é capaz de realizar por meia hora ou mais. Você também pode escolher um valor entre as diferentes atividades. 
Tabela 5

Etapas da equivalência semântica e versão final: Physical Activity Rating (PA-R).

Título T1 Selecione o número apropriado (0 a 7) que melhor descreve seu NÍVEL DE ATIVIDADE FÍSICA no ÚLTIMO MÊS (VF) T2 Use o número adequado (0 a 7) que melhor descreve o seu NÍVEL DE ATIVIDADE no ÚLTIMO MÊS

Escores 0 e 1 T1 NÃO PRATICOU REGULARMENTE ESPORTES NO SEU HORÁRIO DE LAZER OU ATIVIDADES FÍSICAS INTENSAS

0 - Evita caminhar ou se esforçar. Por exemplo, sempre usa o elevador, usa o carro sempre que possivel em vez de caminhar

1 - Caminha por prazer, normalmente usa escadas, às vezes se exercita a ponto de ficar ofegante e suar (VF)

T2 NÃO PARTICIPOU DE FORMA REGULAR EM UM PROGRAMA DE ESPORTES RECREACIONAL OU DE ATIVIDADE FÍSICA INTENSA

0 - Evita caminhar ou se esforçar, por exemplo, sempre usa o elevador, usa o carro sempre que possível em vez de caminhar

1 - Caminha por prazer, normalmente usa escadas, às vezes se exercita a ponto de ficar ofegante e transpirar

Escores 2 e 3 T1 NORMALMENTE O SEU TRABALHO ENVOLVE ATIVIDADES FÍSICAS DE INTENSIDADE MODERADA, OU VOCÊ AS PRATICA NO SEU HORÁRIO DE LAZER, COMO GOLFE, MONTAR A CAVALO, GINÁSTICA, TÊNIS DE MESA, BOLICHE, MUSCULAÇÃO E ATIVIDADES NO QUINTAL

2 - 10 a 60 minutos por semana

3 - Mais de uma hora por semana

T2 NORMALMENTE PARTICIPA DE ATIVIDADES NÃO FORMAIS, OU DE TRABALHO, QUE ENVOLVEM MODERADA ATIVIDADE FÍSICA, COMO GOLFE, MONTAR A CAVALO, GINÁSTICA, TÊNIS DE MESA, BOLICHE, MUSCULAÇÃO E ATIVIDADES NO QUINTAL

2 - 10 a 60 minutos por semana

3 - Mais de uma hora por semana

VF NORMALMENTE O SEU TRABALHO ENVOLVE ATIVIDADES FÍSICAS DE INTENSIDADE MODERADA, OU VOCÊ AS PRATICA NO SEU HORÁRIO DE LAZER, COMO CAMINHAR PARA EXERCITAR-SE, GINÁSTICA, HIDROGINÁSTICA, MUSCULAÇÃO, GOLFE E ATIVIDADES NO QUINTAL

2 - 10 a 60 minutos por semana

3 - Mais de uma hora por semana

Escores 4 e 7 T1 PRATICA REGULARMENTE EXERCÍCIOS FÍSICOS INTENSOS COMO CORRIDA OU "COOPER", NATAÇÃO, CICLISMO, REMO, PULAR CORDA, CORRIDA ESTACIONÁRIA, OU PRATICA ATIVIDADES AERÓBIAS INTENSAS COMO TÊNIS, BASQUETEBOL OU HANDEBOL

4 - Corre menos que uma milha por semana ou pratica atividade comparável por menos de 30 minutos por semana

5 - Corre de 1 a 5 milhas por semana ou pratica atividade comparável de 30 a 60 minutos por semana

6 - Corre de 5 a 10 milhas por semana ou pratica atividade comparável de 1 a 3 horas por semana

7 - Corre mais do que 10 milhas por semana ou pratica atividade comparável por mais de 3 horas por semana

T2 PRATICA REGULARMENTE EXERCÍCIO FÍSICO PESADO COMO CORRIDA OU "COOPER", NATAÇÃO, CICLISMO, REMO, PULAR CORDA, CORRIDA NO MESMO LUGAR, OU PRATICA EXERCÍCIOS MAIS INTENSOS QUE ENVOLVEM ATIVIDADES AERÓBIAS COMO TÊNIS, BASQUETEBOL OU HANDEBOL

4 - Corre menos que 1 milha ou pratica atividade comparável por menos de 30 minutos por semana

5 - Corre 1 a 5 milhas por semana ou gasta 30 a 60 minutos por semana em atividade física comparável

6 - Corre 5 a 20 milhas por semana ou gasta 1 a 3 horas por semana em atividade física comparável

7 - Corre mais de 10 milhas por semana ou gasta mais de 3 horas por semana em atividade física comparável

VF PRATICA REGULARMENTE EXERCÍCIOS FÍSICOS INTENSOS COMO CORRIDA OU "COOPER", NATAÇÃO, CICLISMO, REMO,

PULAR CORDA, FUTEBOL, VOLEIBOL, TÊNIS, BASQUETEBOL OU HANDEBOL

4 - Corre menos que 1,5Km por semana ou pratica atividade comparável por menos de 30 minutos por semana

5 - Corre de 1,5 a $8 \mathrm{~km}$ semana ou pratica atividade comparável de 30 a 60 minutos por semana

6 - Corre de 8 a $16 \mathrm{~km}$ por semana ou pratica atividade comparável de 1 a 3 horas por semana

7 - Corre mais do que $16 \mathrm{~km}$ por semana ou pratica atividade comparável por mais de 3 horas por semana

satisfatória, permitindo que as escalas possam ser aplicadas na população-alvo. Durante todo o processo de adaptação não pareceu haver grandes limitações na adequação dos conceitos apresentados por elas, ou mesmo nas suas formas de apresentação e tradução para o novo idioma.

Com relação à equivalência semântica, o presente estudo não utilizou tradutores nativos do país onde as escalas foram desenvolvidas, o que pode ser considerado uma limitação, uma vez que esta é uma estratégia comum adotada em outros estudos. Contudo, acredita-se que a preocupação com todos os processos subseqüentes (retradução, análise das versões retraduzidas pelos autores originais), além do fato de que as escalas não apresentavam complexidade para uma 
NÃO PRATICOU REGULARMENTE ESPORTES NO SEU HORÁRIO DE LAZER OU ATIVIDADES FÍSICAS INTENSAS

$0 \quad$ Evita caminhar ou se esforçar. Por exemplo, sempre usa o elevador, usa o carro sempre que possivel ao invés de caminhar

$1 \quad$ Caminha por prazer, normalmente usa escadas, às vezes se exercita a ponto de ficar ofegante e suar

NORMALMENTE o seu trabalho envolve ATIVIDADES FÍSICAS DE INTENSIDADE MODERADA, ou você as pratica NO SEU HORÁRIO DE LAZER, COMO CAMINHAR PARA EXERCITAR-SE, GINÁSTICA, HIDROGINÁSTICA, MUSCULAÇÃO, GOLFE, E ATIVIDADES NO QUINTAL

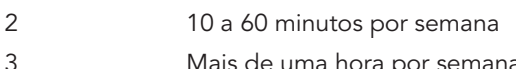

PRATICA REGULARMENTE EXERCÍCIOS FÍSICOS INTENSOS COMO CORRIDA OU "COOPER", NATAÇÃO, CICLISMO, REMO, PULAR CORDA, FUTEBOL, VOLEIBOL, TÊNIS, BASQUETEBOL OU HANDEBOL

$4 \quad$ Corre menos que 1,5km por semana ou pratica atividade comparável por menos de 30 minutos por semana

$5 \quad$ Corre de 1,5 a $8 \mathrm{~km}$ por semana ou pratica atividade comparável de 30 a 60 minutos por semana

$6 \quad$ Corre de 8 a $16 \mathrm{~km}$ por semana ou pratica atividade comparável de 1 a 3 horas por semana

$7 \quad$ Corre mais do que $16 \mathrm{~km}$ por semana ou pratica atividade comparável

Nota: selecione o número apropriado (0 a 7) que melhor descreve seu NÍVEL DE ATIVIDADE FÍSICA no ÚLTIMO MÊS.

Tabela 7

Descrição dos escores das escalas e resultados da avaliação da confiabilidade teste-reteste $(\mathrm{N}=12)$.

\begin{tabular}{lcccc}
\hline Variável & Média (desvio-padrão) & Mínimo-máximo & CCL & IC95\% \\
\hline VSAQ1 & $4,8(1,6)$ & $3-7$ & 0,88 & $0,74-1,00$ \\
VSAQ2 & $4,9(1,7)$ & $2-7$ & & 0,88 \\
RPC1 & $5,2(0,9)$ & $4-8$ & 0,78 & $0,55-1,00$ \\
RPC2 & $5,3(0,9)$ & $5-8$ & $0,08-0,96$ \\
PA-R1 & $2,7(0,9)$ & $0-3$ & 0,91 & $0,89-0,94$ \\
PA-R2 & $2,8(0,6)$ & $1-3$ & & $0,70-0,93$ \\
\end{tabular}

VSAQ: Veterans Specific Activity Questionnaire; RPC: Rating of Perceived Capacity; PAC-R: Physical Activity Rating; CCL: coeficientes de concordância de Lin; $\mathrm{CCl}$ : coeficiente de correlação intraclasse.

adaptação para língua portuguesa, minimizaram o problema. A participação dos autores originais foi importante para uma adaptação adequada e equivalente, pois pelas versões retraduzidas eles confirmaram que elas haviam mantido o significado e o conteúdo das questões presentes nas versões originais.

Uma outra característica importante referese às adaptações feitas para a versão final. Deve-se lembrar que, como o Brasil é um país de grandes diferenças culturais e sociais, talvez outras alterações devam ser feitas de acordo com as características regionais, especialmente na escolha dos exemplos de atividades físicas que se encontram no PA-R. Os dados presentemente relatados são baseados em respostas de indivíduos idosos residentes no Município do Rio de Janeiro, com baixo a médio nível sócio-cultural. Aconselha-se que as versões aqui desenvolvidas sejam mais utilizadas com o intuito de consolidar a adaptação transcultural.

Quanto à equivalência operacional, houve uma limitação: os estudos originais forneceram poucas informações sobre a forma de aplicação das escalas. Isso é essencial para que a metodologia utilizada possa ser repetida e sua ausência denota pouca atenção e preocupação com procedimentos dessa ordem. No presente estudo, percebeu-se certa dificuldade por parte dos indivíduos idosos em preencher as escalas, sem que a mesma fosse lida por um aplicador, fato que fez com que se alterasse sua forma de aplicação. Deve-se evitar, então, que este procedimento interfira nas respostas dos entrevistados.

As propriedades psicométricas das novas versões das escalas foram analisadas, apesar de a equivalência de mensuração ter sido prejudicada. Em nenhuma das escalas originais havia sido 
realizado estudo de reprodutibilidade, situação que impediu comparações entre as versões originais e adaptadas. Os resultados sugerem uma boa reprodutibilidade das escalas, com exceção do RPC. Embora o tamanho da amostra de indivíduos não permita conclusões definitivas sobre as análises de confiabilidade das escalas, alguns comentários podem ser feitos: a menor reprodutibilidade do RPC talvez se deva à própria estrutura do instrumento original, já que é a única das três escalas que não apresenta atividades escritas em cada escore, por exemplo, respondentes que optaram pelo escore de número 5, em um segundo momento (14 dias depois), optaram pelo escore de número 8. Não há opções escritas entre os escores 6 e 7 , o que pode ter induzido a uma maior variação na segunda reposta em comparação com as outras escalas.

\section{Resumo}

O objetivo deste estudo foi realizar a equivalência transcultural de escalas empregadas para a avaliação do nível de atividade física e que são utilizadas como estimativas da aptidão cardiorrespiratória, para posterior utilização em indivíduos idosos. Três escalas foram determinadas após revisão sistemática: Veterans Physical Activity Questionnaire (VSAQ), Rating of Perceived Capacity (RPC) $e$ Physical Activity Rating (PA-R). Para a análise da equivalência, utilizou-se o modelo proposto por Herdman et al. Como parte do processo, a confiabilidade teste-reteste foi avaliada em uma amostra composta por 12 idosos com idade de $74,5 \pm 3,5$ anos, pelo cálculo dos coeficientes de concordância de Lin (CCL) e de correlação intraclasse (CCI). Detectou-se uma boa reprodutibilidade das escalas com exceção do RPC. Apesar de a quantidade de indivíduos não permitir conclusões mais aprofundadas, os resultados podem indicar necessidade de mudanças na estrutura de algumas escalas originais. Por fim, acredita-se que os resultados obtidos no presente estudo sugerem a adequação das versões das escalas para a língua portuguesa, havendo, todavia, a necessidade de um estudo de validade de critério das escalas.

Aptidão Física; Saúde do Idoso; Reprodutibilidade dos Testes
Em suma, acredita-se que os resultados obtidos no presente estudo sugerem que o processo de adaptação transcultural das versões das escalas VSAQ, RPC e PA-R para a língua portuguesa foi adequado. Contudo, para que essas escalas possam ser utilizadas no desenvolvimento de equações sem exercício, há a necessidade da análise de sua validade de critério. Em uma segunda etapa, isso deverá ser feito mediante comparação com os resultados de testes cardiopulmonares de exercício com intensidade progressiva, com mensuração direta do consumo máximo de oxigênio obtido durante o esforço $\left(\mathrm{VO}_{2}\right.$ pico). O resultado obtido deverá ser correlacionado aos escores das novas versões das escalas e, depois, comparado aos escores obtidos pelas escalas originais.

\section{Colaboradores}

G. A. Maranhão Neto foi responsável pela escolha do tema, revisão dos instrumentos, metodologia, análise estatística e elaboração do texto. A. C. M. Ponce de Leon colaborou na análise estatística e revisão do texto. P. T.V. Farinatti contribuiu na revisão e elaboração do texto.

\section{Agradecimentos}

Pesquisa parcialmente financiada pelo Conselho Nacional de Desenvolvimento Científico e Tecnológico (CNPq), sob a forma de bolsa de produtividade em pesquisa para P. T.V. Farinatti (processo no ${ }^{\circ}$ 303018/2003-8). Os autores agradecem ao Dr. Michael E. Reichenheim pelas valiosas contribuições à metodologia do estudo e ao Dr. Vitor Agnew Lira e Bruno Maciel pela contribuição no processo de tradução. 


\section{Referências}

1. Bouchard C, Shephard RJ, Stephens T. Physical activity, fitness, and health: International proceedings and consensus statement. Champaign: $\mathrm{Hu}-$ man Kinetics; 1994.

2. Pate RR, Pratt M, Blair SN, Haskell WL, Macera CA, Bouchard C, et al. Physical activity and public health. A recommendation from the Centers for Disease Control and Prevention and the American College of Sports Medicine. JAMA 1995; 273:402-7.

3. U.S. Department of Health and Human Services. Physical activity and health: a report of the surgeon general. Atlanta: U.S. Department of Health and Human Services, National Center for Chronic Disease Prevention and Health Promotion, Centers for Disease Control and Prevention; 1996.

4. Laukkanen JA, Kurl S, Salonen R, Rauramaa R, Salonen JT. The predictive value of cardiorespiratory fitness for cardiovascular events in men with various risk profiles: a prospective population-based cohort study. Eur Heart J 2004; 25:1428-37.

5. Blair SN, Kohl 3rd HW, Paffenbarger Jr. RS, Clark DG, Cooper KH, Gibbons LW. Physical fitness and all-cause mortality: a prospective study of healthy men and women. JAMA 1989; 262:2395-401.

6. Blair SN, Kampert JB, Kohl 3rd HW, Barlow CE, Macera CA, Paffenbarger Jr. RS, et al. Influences of cardiorespiratory fitness and other precursors on cardiovascular disease and all-cause mortality in men and women. JAMA 1996; 276:205-10.

7. Wei M, Kampert JB, Barlow CE, Nichaman MZ, Gibbons LW, Paffenbarger Jr. RS, et al. Relationship between low cardiorespiratory fitness and mortality in normal-weight, overweight, and obese men. JAMA 1999; 282:1547-53.

8. Ainsworth BE, Richardson MT, Jacobs DR, Leon AS. Prediction of cardiorespiratory fitness using physical activity questionnaire data. Medicine, Exercise, Nutrition and Health 1992; 1:75-82.

9. Mathews CE, Heil DP, Freedson PS, Pastides H. Classification of cardiorespiratory fitness without exercise testing. Med Sci Sports Exerc 1999; 31:486-93.

10. Tammelin T, Nayha S, Rintamaki H. Cardiorespiratory fitness of males and females of Northern Finland Birth Cohort of 1966 at age 31. Int J Sports Med 2004; 25:547-52.

11. D'Alonzo K, Marbach K, Vincent L. A comparison of field methods to assess cardiorespiratory fitness among neophyte exercisers. Biol Res Nurs 2006; 8:7-14.

12. Kohl HW, Blair SN, Paffenbarger Jr. RS, Macera CA, Kronenfeld JJ. A mail survey of physical activity habits as related to measured physical fitness. Am J Epidemiol 1988; 127:1228-39.

13. Miller RA. Kleemeier award lecture: are there genes for aging? J Gerontol A Biol Sci Med Sci 1999; 54: B297-307.

14. Kallinen M. Cardiovascular benefits and potential hazards of physical exercise in elderly people. Journal of Sports Science and Medicine 2005; 4 Suppl 7:1-51.
15. Goraya TY, Jacobsen SJ, Pellikka PA, Miller TD, Khan A, Weston SA, et al. Prognostic value of treadmill exercise testing in elderly persons. Ann Intern Med 2000; 132:862-70.

16. Cherubini A, Lowenthal DT, Williams LS, Maggio D, Mecocci P, Senin U. Physical activity and cardiovascular health in the elderly. Aging 1998; 10:13-25.

17. Hollenberg M, Ngo LH, Turner D, Tager IB. Treadmill exercise testing in an epidemiologic study of elderly subjects. J Gerontol A Biol Sci Med Sci 1998; 53:B259-67.

18. Gill TM, DiPietro L, Krumholz HM. Role of exercise stress testing and safety monitoring for older persons starting an exercise program. JAMA 2000; 284:342-49.

19. Greig C, Butler F, Skelton D, Mahmud S, Young A. Treadmill walking in old age may not reproduce the real life situation. J Am Geriatr Soc 1993; 41: 15-8.

20. Huggett DL, Connelly DM, Overend TJ. Maximal aerobic capacity testing of older adults: a critical review. J Gerontol A Biol Sci Med Sci 2005; 60: 57-66.

21. Simonsick EM, Montgomery PS, Newman AB, Bauer DC, Harris T. Measuring fitness in healthy older adults: the Health ABC Long Distance Corridor Walk. J Am Geriatr Soc 2001; 49:1544-8.

22. Vivacqua R, Serra S, Macaciel R, Miranda M, Bueno N, Campos A. Teste ergométrico em idosos. Parâmetros clínicos, metabólicos, hemodinâmicos e eletrocardiográficos. Arq Bras Cardiol 1997; 68: 9-12.

23. Jackson AS, Blair SN, Mahar MT, Wier LT, Ross RM, Stuteville JE. Prediction of functional aerobic capacity without exercise testing. Med Sci Sports Exerc 1990; 22:863-70.

24. Jurca R, Jackson AS, LaMonte MJ, Morrow Jr. JR, Blair SN, Wareham NJ, et al. Assessing cardiorespiratory fitness without performing exercise testing. Am J Prev Med 2005; 29:185-9.

25. Myers J, Do D, Herber W, Ribisl P, Froelicher VF. A nomogram to predict exercise capacity from a specific questionnaire and clinical data. Am J Cardiol 1994; 73:591-6.

26. Myers J, Bader D, Madhavan R, Froelicher V. Validation of a specific activity questionnaire to estimate exercise tolerance in patients referred for exercise testing. Am Heart J 2001; 142:1041-6.

27. Guillemin F. Cross-cultural adaptation and validation of health status measures. Scand J Rheumatol 1995; 24:61-3.

28. Maranhão Neto GA, Lourenço PMC, Farinatti PTV. Equações de predição da aptidão cardiorrespiratória sem testes de exercício e sua aplicabilidade em estudos epidemiológicos: uma revisão sistemática. Cad Saúde Pública 2004; 20:48-56.

29. Maeder M, Wolber T, Atefy R, Gadza M, Ammann P, Myers J, et al. Impact of the exercise mode on exercise capacity: bicycle testing revisited. Chest 2005; 128:2804-11.

30. Wisen AG, Farazdaghi RG, Wohlfart B. A novel rating scale to predict maximal exercise capacity. Eur J Appl Physiol 2002; 87:350-7. 
31. Mathews CE, Heil DP, Freedson PS, Pastides H. Classification of cardiorespiratory fitness without exercise testing. Med Sci Sports Exerc 1999; 31:486-93.

32. Heil DP, Freedson PS, Ahlquist LE, Price J, Rippe J. Nonexercise regression models to estimate peak oxygen consumption. Med Sci Sports Exerc 1995; 27:599-606.

33. George JD, Stone WJ, Burkett LN. Non-exercise VO2max estimation for physically active college students. Med Sci Sports Exerc 1997; 29:415-23.

34. Wier LT, Jackson AS, Ayers GW, Arenare B. Nonexercise models for estimating VO2max with waist girth, percent fat, or BMI. Med Sci Sports Exerc 2006; 38:555-61.
35. Bradshaw DI, George JD, Hyde A, LaMonte MJ, Vehrs PR, Hager RL, et al. An accurate VO2max nonexercise regression model for 18-65-year-old adults. Res Q Exerc Sport 2005; 76:426-32.

36. Herdman M, Fox-Rushby J, Badia X. A model of equivalence in the cultural adaptation of HRQoL instruments: the universalist approach. Qual Life Res 1998; 7:323-35.

37. Lin LI. A concordance correlation coefficient to evaluate reproducibility. Biometrics 1989; 45: 255-68.

38. Sánchez R, Echeverry J. Validación de escalas de medición en salud. Rev Salud Pública 2004; 6: 302-18.

Recebido em 28/Nov/2007

Aprovado em 16/Abr/2008 